\title{
COMMUTATIVE REGULAR RINGS WITHOUT PRIME MODEL EXTENSIONS
}

\author{
D. SARACINO ${ }^{1}$ AND V. WEISPFENNING ${ }^{2}$
}

ABSTRACT. It is known that the theory $K$ of commutative regular rings with identity has a model completion $K^{\prime}$. We show that there exists a countable model of $K$ which has no prime extension to a model of $K^{\prime}$.

If $K$ and $K^{\prime}$ are theories in a first order language $L$, then $K^{\prime}$ is said to be a model completion of $K$ if $K^{\prime}$ extends $K$, every model of $K$ can be embedded in a model of $K^{\prime}$, and for any model $A$ of $K$ and models $B_{1}, B_{2}$ of $K^{\prime}$ extending $A$, we have $\left\langle B_{1} \underline{a}\right\rangle_{a \in A} \equiv\left\langle B_{2}, \underline{a}\right\rangle_{a \in A}$, i.e. $B_{1}$ and $B_{2}$ are elementarily equivalent in a language which has constants for the elements of $A$. If a theory $K$ has a model completion $K^{\prime}$, then the models of $K^{\prime}$ can reasonably be regarded as the "algebraically closed" models of $K$; for example, the theory of algebraically closed fields is the model completion of the theory of fields. It was shown in [3] that the theory $K$ of commutative regular rings with identity (formulated in the usual language $L$ for rings with identity) has a model completion. We recall that a commutative ring $R$ with identity is said to be regular (in the sense of von Neumann) if for any element $a \in R$ there exists $b \in R$ such that $a^{2} b=a$. (A good reference is Lambek [2].) The model completion $K^{\prime}$ is given by the following axioms:

(i) the axioms of commutative regular rings with identity;

(ii) an axiom stating that there are no minimal idempotents, i.e.

$$
\forall x\left(x^{2}=x \wedge x \neq 0 \rightarrow \exists y\left(y^{2}=y \wedge y \neq 0 \wedge y \neq x \wedge y x=y\right)\right) ;
$$

(iii) a set of axioms stating that every monic polynomial has a root.

Received by the editors July 30, 1973 and, in revised form, December 21, 1973. AMS (MOS) subject classifications (1970). Primary 02H13, 02H15; Secondary 13L05, $13 \mathrm{~B} 99$.

Key words and phrases. Model completion, commutative regular ring, prime model extension, finite forcing.

1 Research supported in part by NSF Grant GP 34088.

2 Research supported in part by Deutsche Forschungsgemeinschaft. 
Wé will also consider the theory $K^{*}$ obtained by deleting axiom (ii) from $K^{\prime}$.

If $B$ is a model of a theory $T$, and $A$ is a substructure of $B$, then $B$ is called a prime model extension (for $T$ ) of $A$, if for every model $C$ of $T$ extending $A$, there exists an embedding $f: B \rightarrow C$ which is the identity on $A$. Of course if $T$ is model-complete (as in $K^{\prime}$ ), then "embedding" can be replaced by "elementary embedding". In this paper we consider the question of whether over every commutative regular ring $A$ there exists a prime model extension for $K^{\prime}$; we also consider the same question for $K^{*}$. In both cases the answer is negative.

We begin by recalling some model theoretic preliminaries in the setting of commutative regular rings. Let $R$ be a model of $K$; we expand $L$ to the language $L(R)$ by adding a new constant symbol $\underline{a}$ for every element $a \in R$. The diagram $D(R)$ of $R$ is the set of all polynomial equations and in equations involving the constants $\underline{a}$ which hold in $R$ when $\underline{a}$ is interpreted as $a$. Every model of the theory $K^{\prime} \cup D(R)$ is (up to an isomorphism) an extension of $R$.

Let $F(R)$ be the set of formulas in $L(R)$ with one free variable $x$. For $\phi, \psi$, in $F(R)$ we set $\phi \sim \psi$, if and only if $K^{\prime} \cup D(R) \vdash \phi \leftrightarrow \psi$. This gives us an equivalence relation on $F(R)$; we denote the equivalence class of $\phi$ by $[\phi]$. The equivalence classes form a Boolean algebra $B(R)$ (called the Lindenbaum algebra of $K^{\prime} \cup D(R)$ ) under the operations $[\phi]+[\psi]=[\phi \vee \psi]$, $\left.[\phi]^{\prime}=[\rceil \phi\right]$. We call the elements of the Stone space $S(R)$ of $B(R) 1$-types over $R$. Notice that a point $p \in S\left(R^{\prime}\right)$ is isolated in the Stone topology if and only if there is a formula $\phi$ such that $K^{\prime} \cup D(R) \vdash \phi \rightarrow \psi$ for every $\psi$ such that $[\psi] \in p$, and $[\phi] \neq 0$ in $B(R)$. Such a formula $\phi$ is called a generator for $p$.

Since $K^{\prime} \cup D(R)$ is complete, it is clear that if $p \in S(R)$ is isolated, then every model $A$ of $K^{\prime} \cup D(R)$ realizes $p$, i.e. there exists $a \in A$ such that $\{[\phi]: A \vDash \phi(a)\}=p$.

We shall present our results within the framework of finite forcing relative to $K \cup D(R)$ (for $R$ countable). The fundamental papers on finite forcing in model theory are [1] and [4]. We expand $L(R)$ to $L(R, C)$ by adding a countable set $C$ of new constant symbols. In our setting, a forcing condition $q\left(\underline{a}_{1}, \cdots, \underline{a}_{m}, \underline{c}_{1}, \cdots, \underline{c}_{n}\right)$ is a finite set of polynomial equations and inequations in the language $L(R, C)$ which is consistent with $K \cup D(R)$, i.e. such that there exists a model $A$ of $K$ extending $R$ and $c_{1}, \cdots, c_{n}$ in $A$ such that $A$ satisfies all the statements in $q$ when each $\underline{a}_{i}$ is interpreted as $a_{i}$, and each $\underline{c}_{i}$ is interpreted as $c_{i *}$ If $q$ is a condition and $\phi$ is a 
sentence in $L(R, C)$, then the notion " $q$ forces $\phi$ " is defined by induction on the structure of $\phi$, as follows:

(i) If $\phi$ is an equation, $q$ forces $\phi$ if and only if $\phi \in q$;

(ii) $q$ forces $\phi \wedge \psi$ if and only if $q$ forces $\phi$ and $q$ forces $\psi$;

(iii) $q$ forces $\phi \vee \vee \psi$ if and only if $q$ forces $\phi$ or $q$ forces $\psi$ or both;

(iv) $q$ forces $\exists x \phi(x)$ if and only if for some $\underline{c} \in C, q$ forces $\phi(\underline{c})$;

(v) $q$ forces $\urcorner \phi$ if and only if for no condition $q^{\prime}$ extending $q$. is it the case that $q^{\prime}$ forces $\phi$.

A sequence $\left\{q_{i}\right\}_{i=1}^{\infty}$ of conditions is complete if for each sentence $\phi$ of $L(R, C)$ there is some $q_{i}$ which forces either $\phi$ or $\urcorner \phi$. A complete sequence determines in a canonical way (see [1] or [4]) a ring $A$ which contains $R$. Every element of $A$ is named by some $\underline{c}$ in such a way that all the statements in any $q_{i}$ hold in $A$ (when $\underline{a}$ is interpreted as $a$ for $a \in R$ ), and any equation which holds in $A$ is in some $q_{i}$. $A$ is called finitely generic for $K \cup D(R)$. Since $K \cup D(R)$ has a model completion (namely $K^{\prime} \cup$ $D(R)$ ), this implies [1] that $A$ is a model of $K^{\prime} \cup D(R)$; in particular $A$ is a model of $K^{\prime}$.

We can now prove

Theorem 1. There exists a countable model $R$ of $K$ which has no prime extension to a model of $K^{\prime}$. Moreover, $R$ can be chosen so that all the isolated points in $S(R)$ are realized in $R$, i. e. have a generator $x=\underline{r}$ for some $r \in R$. In particular the isolated points are not dense in $S(R)$.

Corollary. $K^{\prime}$ is not quasi-totally transcendental. (For this notion see [5].)

Remark. It is easy to see that $K^{\prime}$ is $K$-unstable for all infinite cardinals $\kappa$.

Proof of Theorem 1. Let $R^{\prime}$ be the ring of all locally constant functions from the Cantor space $X$ into $\bar{Q}$, an algebraic closure of the rationals $Q$. (A function $f$ on $X$ is locally constant if for every $x \in X$ there exists a neighborhood $U$ of $x$ on which $f$ is constant.) $R^{\prime}$ is a model of $K^{\prime}$ and $R^{\prime}$ is countable. For, notice that any locally constant function $f$ on $X$ determines a partition of $X$ into finitely many clopen sets $P_{i}$ such that $f$ is constant on each $P_{i}$. Since $\bar{Q}$ is countable, any such partition is determined in this way by only countably many $f$ 's; and there are only countably many such partitions of $X$.

Pick a point $x_{0} \in X$. Let $R \subset R^{\prime}$. consist of all the elements $f \in R^{\prime}$ such that $f\left(x_{0}\right) \in Q$. It is easy to see that $R$ is a regular ring. 
Now suppose $R$ has a prime extension $M$ to a model of $K^{\prime}$. We can assume $R \subseteq M \subseteq R^{\prime}$, and then we know that $M$ is an elementary substructure of $R^{\prime}$. Since $R$ is not a model of $K^{\prime}$, we can pick $d \in M-R$.

Let $p$ be the point of $S(R)$ realized by $d$ in $M$. We will show that there exists a model of $K^{\prime} \cup D(R)$, no element of which realizes $p$; it follows that $M$ cannot be embedded in this model over $R$, so $M$ is not prime.

Let $C$, as before, be a countable set of new constants not in $L(R)$, and let $\left\{\phi_{i}\right\}_{i=1}^{\infty}$ be an enumeration of the sentences in $L(R, C)$. We will in a moment define a complete sequence $\left\{q_{i}\right\}$ of forcing conditions, but first we state the following

Lemma. Let $q$ be a condition. Let $\underline{c}_{1}, \cdots, \underline{c}_{n}$ be the elements of $C$ mentioned in $q$. Then there exist elements $a_{1}, b_{1}, \cdots, a_{n}, b_{n}$ in $R$ such that $q \cup\left\{\underline{b}_{i} \neq \underline{c}_{i} \underline{a}_{i} \mid i=1, \cdots, n\right\}$ is a condition, where $a_{i}, b_{i} \in R$ are such that $b_{i}=d a_{i}$ in $R^{\prime}$.

We will prove the Lemma later.

Now define a sequence $\left\{q_{i}\right\}$ of conditions as follows: Let $q_{1}$ be a condition which forces either $\phi_{1}$ or $7 \phi_{1}$. Let $q_{2}$ be an extension of $q_{1}$ obtained by using the Lemma. If $i>1$ is odd, let $q_{i}$ be an extension of $q_{i-1}$ which forces either $\phi_{(i+1) / 2}$ or $7 \phi_{(i+1) / 2}$. If $i>1$ is even, let $q_{i}$ be an extension of $q_{i-1}$ obtained by using the Lemma. As indicated above, the complete sequence $\left\{q_{i}\right\}$ determines a model $R^{\prime \prime}$ of $K^{\prime}$ which extends $R$, and every element of $R^{\prime \prime}$ is denoted by some $\underline{c} \in C$.

Suppose some element $r \in R^{\prime \prime}$ realizes $p$, and that this element is denoted by $\underline{c}_{i}$. Then for some odd $j, \underline{c}_{i}$ appears in $q_{j}$. Thus there exist $a_{i}$, $b_{i}$ in $R$ such that $b_{i}=d a_{i}$ in $R^{\prime}$ and $q_{j+1}$ contains the statement $\underline{b}_{i} \neq$ $\underline{c}_{i} \underline{a}_{i}$. Since $\underline{b}_{i} \neq \underline{c}_{i} \underline{a}_{i}$ is in $q_{j+1}, \underline{b}_{i} \neq x \underline{a}_{i}$ is in the type realized by $r$ in $R^{\prime \prime}$. But $\underline{b}_{i}=x \underline{a}_{i}$ is in $p$, since $p$ is the type realized by $d$ in $R^{\prime}$ and $b_{i}=d a_{i}$. Thus $r$ does not realize $p$ in $R^{\prime \prime}$. This proves the first statement of the theorem.

To prove the second statement, suppose there is an isolated point $p$ in $S(R)$ which is not realized in $R$. Since $p$ is isolated there is $d$ in $R^{\prime}-$ $R$ which realizes $p$. Then by the above, there exists a model of $K^{\prime} \cup D(R)$, no element of which realizes $p$. Hence $p$ is not isolated, a contradiction.

To see that the isolated points are not dense in $S(R)$, observe that if $\phi(x)$ is a formula in $L(R)$ such that $K^{\prime} \cup D(R) \vdash \exists x \phi(x)$, but $\left.R \models\right\rceil \exists x \phi(x)$, then the neighborhood in $S(R)$ determined by $\phi(x)$ contains no isolated 
points, since all the isolated points are realized in $R$. An example of such a formula is $x^{2}=2$.

This finishes the proof, modulo the Lemma.

In proving the Lemma we will work with idempotents of $R^{\prime}$, and it will be helpful to make some preparatory remarks about them. An idempotent $e$ of $R^{\prime}$ is an element $e$ in $R^{\prime}$ whose values are everywhere either 0 or 1 . We identify $e$ with the clopen subset of $X$ consisting of all points $x \in X$ such that $e(x)=1$. This gives a 1-1 correspondence between the idempotents of $R^{\prime}$ and the clopen subsets of $X$. Thus if $e, f$ are idempotents, we say $e \subset f$ if $e(x)=1$ implies $f(x)=1$ for all $x \in X$. Furthermore the correspondence makes it clear what we mean when we say that an element of $R^{\prime}$ is constant on some idempotent.

Let $q$ be a condition, let $h_{1}, \cdots, h_{m}$ be all the elements of $R$ such that $\underline{b}_{i}$ occurs in $q$, and let $\underline{c}_{1}, \cdots, c_{n}$ be the elements of $C$ which occur in $q$. Let $\phi\left(x_{1}, \cdots, x_{n}\right)$ be the formula obtained by replacing $c_{i}$ by $x_{i}$ in the conjunction of the elements of $q$. Then since $q$ is a condition, the formula $\exists x_{1} \cdots \quad \exists x_{n} \phi$ holds in some model of $K^{\prime} \cup D(R)$, and hence in all of them, since $K^{\prime}$ is the model completion of $K$. In particular $R^{\prime} \vDash \exists x_{1}$ $\cdots \quad \exists x_{n} \phi$, so there exist elements $c_{1}, \cdots, c_{n}$ in $R^{\prime}$ such that $R^{\prime} \vDash$ $\phi\left(\underline{c}_{1}, \cdots, \underline{c}_{n}\right)$ when $\underline{c}_{i}$ is interpreted as $c_{i}$ and $\underline{b}_{i}$ is interpreted as $h_{i}$. $\phi\left(\underline{c}_{1}, \cdots, \underline{c}_{n}\right)$ is a conjunction of polynomial equations $\left\{P_{i, 1}=P_{i, 2}\right\}_{i=1}^{s}$ and inequations $\left\{P_{s+i, 1} \neq P_{s+i, 2}\right\}_{i=1}^{t}$ which hold in $R^{\prime}$. For each $i, 1 \leq i$ $\leq t$, let $x_{i} \neq x_{0}$ be a point in $X$ at which $P_{s+i, 1} \neq P_{s+i, 2}$. Let $e$ be an idempotent of $R$ such that $e\left(x_{0}\right)=1$ and $e\left(x_{i}\right)=0$ for $1 \leq i \leq t$. (The fact that $e$ exists follows from the properties of $X$.) Let $f C e$ be an idempotent such that $f\left(x_{0}\right)=1$ and each of $d, h_{1}, \cdots, h_{m}, c_{1}, \cdots, c_{n}$ is constant on $f$. Notice that since each $h_{i} \in R$, each $h_{i}$ has a constant rational value on $f$.

Let $d^{*}$ denote the constant irrational value of $d$ on $f$. Let $u, v$ in $R$ be idempotents such that $u, v \subset f, u\left(x_{0}\right)=v\left(x_{0}\right)=0, u v=0$, and $u \neq 0, v \neq 0$. Let $y, z$ in $R$ be such that $y(x)=d^{*}$ for all $x \in u$ and $y(x)=0$ for all $x \notin u, z(x)=d^{*}$ for all $x \in v, z(x)=0$ for all $x \notin v$.

Since $d^{*}$ is irrational, there exists an automorphism $\rho$ of $\bar{Q}$ such that $\rho\left(d^{*}\right) \neq d^{*}$. Denote the constant values of the $h_{i}$ and $c_{i}$ on $f$ by $b_{i}^{*}, c_{i}^{*}$, respectively. Then each $h_{i}^{*} \in Q$, so $\rho\left(h_{i}^{*}\right)=h_{i}^{*}$ for each $i$. Let $c_{i}^{\prime}, 1 \leq i$ $\leq n$, be the element of $R^{\prime}$ which has the constant value $\rho\left(c_{i}^{*}\right)$ on $u$ and the same values as $c_{i}$ on $1-u$.

Let $J=\left\{j \in\{1, \cdots, n\} \mid c_{j}\right.$ has the constant value $d^{*}$ on $\left.f\right\}$. For $i \epsilon$ $J$ let $a_{i}=u$ and let $b_{i}=y$. For $i \notin J$ let $a_{i}=v$ and let $b_{i}=z$. 
We claim that $q \cup\left\{\underline{b}_{i} \neq \underline{c}_{i} \underline{a}_{i} \mid 1 \leq i \leq n\right\}$ holds in $R^{\prime}$ when we interpret $\underline{a}_{i}$ as $a_{i}, \underline{b}_{i}$ as $b_{i}, \underline{b}_{i}$ as $b_{i}$, and $\underline{c}_{i}$ as $c_{i}^{\prime}$. The fact that the part in brackets holds follows immediately from the definition of the $a_{i}, b_{i}$, and $c_{i}^{\prime}$. The inequalities in $q$ hold, because the $c_{i}^{\prime}$ agree with the $c_{i}$ on $x_{1}$, $\cdots, x_{i}$. The equalities hold, because the $c_{i}^{\prime}$ differ from the $c_{i}$ only on $u$, and on $u$ the equalities hold for the $c_{i}^{\prime}$, because $\rho$ is an automorphism of $\bar{Q}$.

This completes the proof.

Remarks. (1) Using the model completeness of $K^{\prime}$, one can argue directly from the Lemma that the type of $d$ is not principal; one can then fall back on the standard omitting types theorem to conclude that $R$ has no prime extension to a model of $K^{\prime}$. However we feel that the presentation in terms of forcing is more intuitive and more nearly self-contained.

(2) In the general framework of [5], one considers theories which are model completions of universal theories.

In any commutative regular ring there exists for any element $x$ a unique element $f(x)$ such that $x^{2} f(x)=x$ and $f(x)^{2} x=f(x)$ (see [2]). If we enlarge our language $L$ to $L^{\prime}$ by adding a 1-place function symbol $f$, and write the axiom of regularity in the form

$$
\forall x\left(x^{2} f(x)=x \wedge f(x)^{2} x=f(x)\right),
$$

then, as is remarked in [3], $K^{\prime}$ is in $L^{\prime}$ the model completion of $K$, and $K$ in $L^{\prime}$ is a universal theory. Since the function $f$ is definable in $L$, it is easy to see that Theorem 1 holds for $L^{\prime}$ as well as for $L$. Thus with respect to $L^{\prime}, K^{\prime}$ is a natural example of a model completion of a universal theory $K$ which has a countable model $R$ such that the isolated points are not dense in $S(R)$.

Recall that $K^{*}$ is $K^{\prime}$ with axiom (ii) deleted.

Theorem 2. If $R$ is the ring of Theorem 1, then $R$ has no prime extension to a model of $K^{*}$.

Proof. Suppose $A$ is a prime extension of $R$ to a model of $K^{*}$. Then since $R^{\prime}$ (as defined in the proof of Theorem 1) is a model of $K^{*}$, there exists an embedding $f: A \rightarrow R^{\prime}$ such that $f \mid R$ is the identity map. Therefore $A$ has the same idempotents as $R$, since $R^{\prime}$ has the same idempotents as $R$. Thus there are no minimal idempotents in $A$, so $A$ is in fact a model of $K^{\prime}$. Thus $A$ is a prime extension of $R$ to a model of $K^{\prime}$, contradicting Theorem 1.

We should point out that $R^{\prime}$ is a minimal extension of $R$ to a model of 
$K^{\prime}$ (and $K^{*}$ ). That is, there is no model of $K^{\prime}$ (or $K^{*}$ ) sitting strictly between $R$ and $R^{\prime}$. For suppose $S$ is a model of $K^{*}$ and $R \subseteq S \subseteq R^{\prime}$. Then for any $x \in X,\{f(x) \mid f \in S\}=\bar{Q}$. Now let $f \in R^{\prime}$; we claim $f \in S$. To see this, we observe that if $x \in X$ there exists an idempotent $e_{x}$ containing $x$ and $f_{x} \in S$ such that $f_{x}$ and $f$ have the same constant value on $e_{x}$. It follows from the properties of $X$ that there exist disjoint idempotents $e_{\underline{1}}$, $\cdots, e_{n}$ in $R$ and elements $f_{1}, \cdots, f_{n}$ in $S$ such that $\Sigma_{i=1}^{n} e_{i}=1$, and $f_{i} e_{i}$ $=f e_{i}, 1 \leq i \leq n$. Then

$$
f=\sum_{i=1}^{n} f e_{i}=\sum_{i=1}^{n} f_{i} e_{i} \in S .
$$

\section{REFERENCES}

1. J. Barwise and A. Robinson, Completing theories by forcing, Ann. Math. Logic 2 (1970), 119-142.

2. J. Lambek, Lectures on rings and $\cdot$ modules, Blaisdell, Waltham, Mass., 1966. MR 34 \#5857.

3. L. Lipshitz and D. Saracino, The model companion of the theory of commutative rings without nilpotent elements, Proc. Amer. Math. Soc. 38 (1973), 381-387.

4. A. Robinson, Forcing in model theory, Symposia Mathematica, vol. V (INDAM, Rome, 1969/70), pp. 69-82. MR 43 \#4651.

5. G. E. Sacks, Saturated model theory, Benjamin, Reading, Mass., 1972.

DEPARTMENT OF MATHEMATICS, YALE UNIVERSITY, NEW HAVEN, CONNECTICUT 06520

Current address (D. Saracino): Department of Mathematics, Colgate University, Hamilton, New York 13346

Current address (V. Weispfenning): Mathematisches Institut der Universität, Heidelberg, West Germany 